\title{
A comparative study of online medical procedure learning via video versus text modalities during the COVID-19 pandemic
}

\author{
Kritsanaviparkporn, C., Kitcharoen, N., Yimcharoen, N., Thaweesuk, S., Maneerit, S., \\ Treesirichod, $A$.
}

\begin{abstract}
Background: Online learning has become the alternative in medical education during the coronavirus2019 (COVID-19) pandemic. We aimed to compare the efficacy of utilizing video and text modalities in knowledge acquisition of a new clinical procedure in medical students during the COVID-19 lockdown.

Methods: The target population were recruited via convenience sampling and allocated by simple randomization. The chosen clinical procedure was knee arthrocentesis, which participants with prior experience were excluded. Participants were instructed to either watch a demonstration video or read a transcribed manuscript once, before answering 10 multiple choice questions on the procedure. Outcome measure was the average test score in each group.
\end{abstract}

Results: Demographic characteristics were comparable between the research arms (video $n=56$, text $\mathrm{n}=59$ ). The median score of the video group (median $=8, I Q R 7-9$ ) was statistically higher than the text group (median $=6$, IQR 4-8) $(p<0.01)$. Higher proportions of correct answers were noted in the video group in each individual questions.

Conclusion: Demonstration video is superior to text-based modality for knowledge acquisition of a new medical procedure. Therefore, the use of video tutorial is encouraged during the COVID-19 online learning phase, to ensure students have a solid knowledge foundation ready for when clerkship resumes.

Keywords: online learning, medical student, knowledge acquisition, COVID-19

\section{Introduction}

Online learning has become one of the main tools for medical students to independently develop their clinical competence (Pai et al., 2014). Ensuring proficient in medical procedures during their time in school ensures students will be able to provide optimal and standardized care for patients in the future (Roy et al., 2018, Komasawa et al., 2020). The Fitts and Posner

Faculty of Medicine, Srinakharinwirot University, Nakhonnayok, Thailand

Corresponding author: Dr Arucha Treesirichod treesar@yahoo.com

DOI: http://doi.org/10.4038/seajme.v15i2.369 model of skill acquisition emphasizes that learning a skill initially involves knowledge acquisition (cognition stage) (Fitts PM, 1979), before it can become a fluent, implicit, and effortless behavior (integration and automation phases) (Taylor and Ivry, 2012, Shaker, 2018). Similarly, according to the Miller's pyramid, competence progresses from 'knows', 'knows how', 'shows', to 'does', whereby advancement through the hierarchy requires refinement of knowledge, skills, and attitude (Miller, 1990). As such, a student may initially watch a demonstration video to develop the 'knows' and 'knows how'. Then, they practice on manikins and eventually perform the skill on patients, reflecting the 'shows' and 'does' (Shaker, 2018,

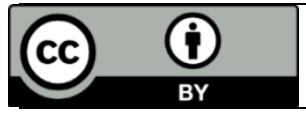

(C) SEAJME. This is an Open Access article distributed under the terms of the Creative Commons Attribution License (http://creativecommons.org/licenses/by/4.0/), which permits unrestricted use, distribution, and reproduction in any medium, provided the original author and source are credited 
Miller, 1990). Therefore, effective knowledge acquisition would ease the student's transition towards a higher degree of competence.

Amidst the coronavirus disease 2019 (COVID19) pandemic, online learning has become the forefront alternative to traditional classroom to ensure continuity of medical education (Sandhu and de Wolf, 2020). This adjustment was only possible due to gadgets and webinar platforms being readily available, allowing students to learn whenever and wherever they desire (O'Doherty et al., 2018, Sandhu and de Wolf, 2020). With a variety of modalities, such as audiovisual clips and interactive texts, educators are able to effectively structure their teachings, while students are free to explore their preferred learning style (Worm and Jensen, 2013).

The choice of online learning modality is one of the essential factors that marks the student for a solid foundation (Worm and Jensen, 2013). For example, the selection of video over illustrative-text resulted in greater knowledge and technical proficiency of the Dix-Hallpike test in Danish medical students (Reck-Burneo et al., 2018). Likewise, surgical trainees who watched operative videos have greater confidence than those who read a manuscript on the procedure (Buch et al., 2014). While the previous studies were conducted prior to COVID-19, the effectiveness of learning and assessing the skill through an entirely onlinebased system remains undetermined. Therefore, we aimed to compare two commonly used modalities, demonstration video and textbased modalities, in knowledge acquisition of a new clinical skill during the COVID-19 online learning phase.

\section{Methods}

This educational research was a two-groups randomized experiment. The target population was preclinical year's medical students from Srinakharinwirot University, Thailand. Participants were voluntarily recruited by convenience sampling and were free to withdraw from the experiment at any moment. Participants were allocated to either video or text group through a simple computergenerated randomization list. They were excluded if they reported previous clinical experience in knee arthrocentesis. The sample size was estimated for alpha (a) and beta ( $\beta$ ) levels of 0.05 and 0.10 , respectively. Based the results from the pilot cohort $(n=40)$ with a $1: 1$ video to text samples, 56 participants were needed in each group. After accounting for a $5 \%$ previous experience in the procedure, the total sample size required was 118 participants. The study protocol was approved by the Institutional Review Board and the Ethics Committee of Srinakharinwirot University.

The chosen procedure was knee arthrocentesis because it is usually taught during the clinical years in the Thai medical curriculum. Therefore, it was assumed that the target group had no background knowledge of this skill. Participants could learn the content once without controlling time, which the video group were instructed not to pause, rewind, or fast-forward the content.

\section{Development of test instrument}

The experiment was conducted via an online Google Form, whereby separate sets were given to each group. The tests consisted of 3 parts, as summarized in Figure 1.

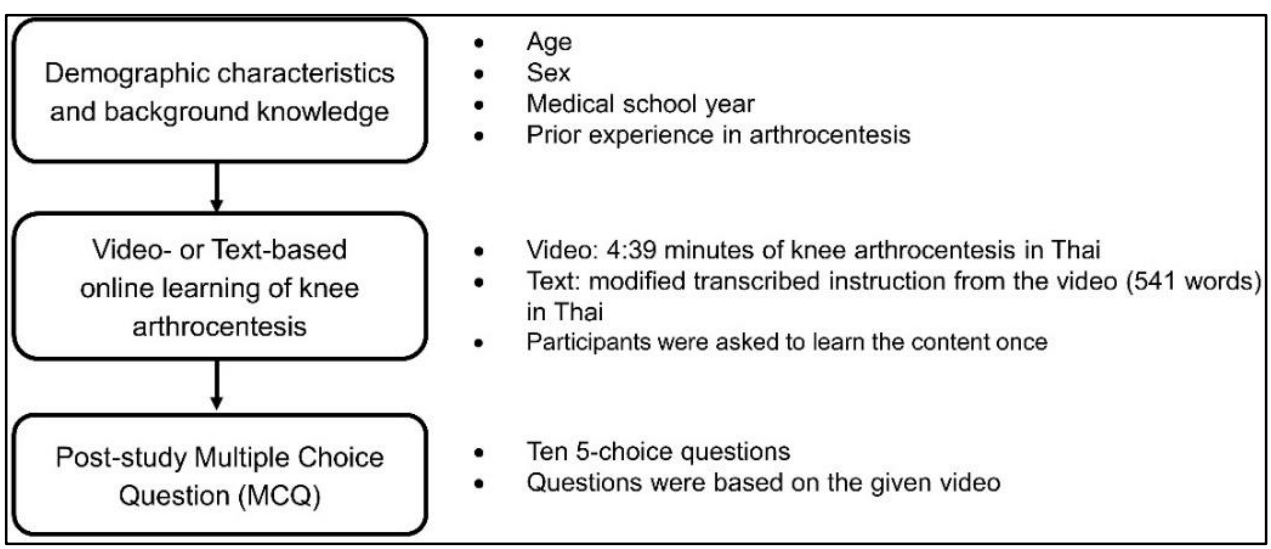

Figure 1: The structure of the experiment

South-East Asian Journal of Medical Education

Vol. 15, no. 2, 2021 
Part 1: Questions on demographic characteristics and prior experience with the procedure: Participants who answered that they had prior experience in knee arthrocentesis were automatically directed to the end of the experiment.

Part 2: The online learning platform, which consisted of either video or text modality: The video was a demonstration of knee arthrocentesis on a manikin in Thai, developed in-house by the Department of Internal Medicine, Srinakharinwirot University. The demonstration addressed the indication, required equipment, steps to aspiration of synovial fluid from the knee, periprocedural care, and complications. The length of the video was 4 minutes and 39 seconds. Its text-based counterpart was a passage transcribed and modified from the video in Thai. Its content validity was verified by three orthopedic professors, with an index of item congruence (IOC) of 1.

Part 3: Assessing knowledge by multiple choice questions (MCQ): The test consisted of ten 5choice MCQs written in Thai on knowledge of knee arthrocentesis. The content validity of the post-study test was assessed via three orthopedic professors with an IOC of 0.80 . Results from the pilot cohort $(n=40)$ demonstrate a reliability measure, assessed via Kuder-Richardon (KR-20), of 0.81, and discrimination and difficulty indices of 0.30 and 0.55 , respectively.

\section{Outcome measurement}

The primary outcome was the average multiplechoice question (MCQ) test score in each group, which was an indicator of knowledge acquisition.

\section{Data Analysis}

For descriptive statistics, gender and proportion of correct answers for each question were presented as percentages. For average MCQ scores, the normality was determined using the D'Agostino-Pearson test. If the collected data were to be normally distributed, the mean and standard deviation would be presented along with a two-tailed independent t-test. Conversely, the median, corresponding interquartile ranges, and Mann Whitney- $U$ test would be calculated if the data were a nonnormal distribution. Results were considered significant when $p<0.05$.

\section{Results}

A total of 118 preclinical years' medical students was recruited, in which 3 were excluded due to previous experience in knee arthrocentesis. Therefore, 56 and 59 participants were randomized to video and text groups, respectively (Figure 2). Gender differences were similar between groups, at which $48.2 \%$ and $49.2 \%$ were male in the video and text cohorts, respectively.

The MCQ scores in the video group was higher (median $=8$, IQR 7-9) than the text $($ median $=$ 6, IQR 4-8) with statistical significance $(p=0.033)$. The video group also consistently demonstrated greater percentages of correct answers in all individual questions, with significant differences observed in Question 2 $(p=0.012)$, Question $8(p=0.010)$, and Question $9(p=0.012)$ (Table 1).

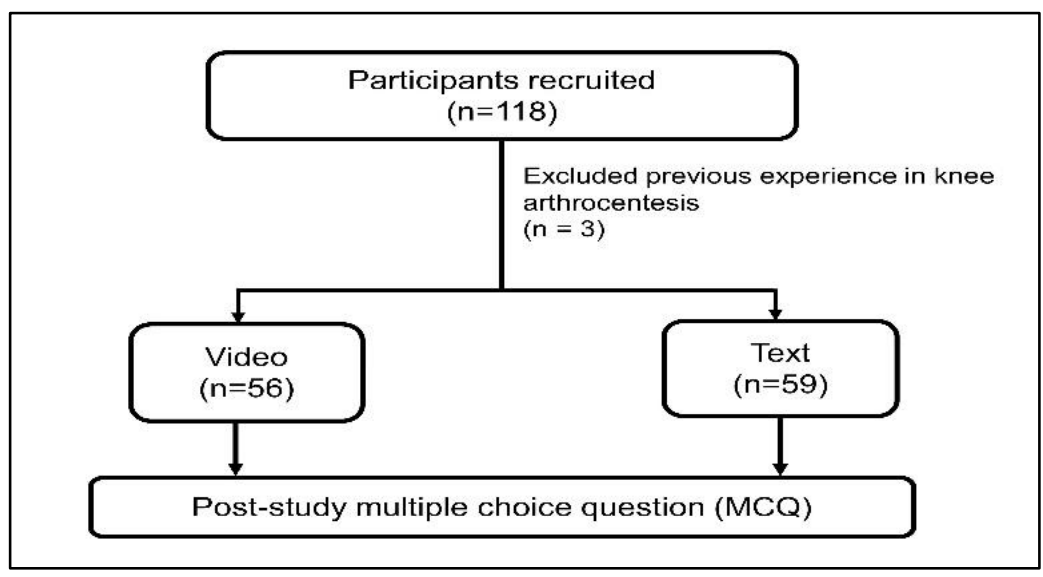

Figure 2: Flowchart of the study sequence

South-East Asian Journal of Medical Education Vol. 15, no. 2, 2021 
Table 1. Frequency of correct answers to each individual question

\begin{tabular}{|c|c|c|c|}
\hline \multirow{2}{*}{ Multiple Choice Questions } & \multicolumn{2}{|c|}{ Frequency of Correct Answers } & \multirow{2}{*}{$p$-value* } \\
\hline & Video $(n=56)(\%)$ & Text $(n=59)(\%)$ & \\
\hline $\begin{array}{l}\text { Q1. Which of the following are the indications for } \\
\text { performing knee arthrocentesis? }\end{array}$ & $44(78.6)$ & $37(62.7)$ & 0.062 \\
\hline $\begin{array}{l}\text { Q2. Which list of equipment should be prepared for knee } \\
\text { arthrocentesis? }\end{array}$ & $32(57.1)$ & $20(33.9)$ & 0.012 \\
\hline $\begin{array}{l}\text { Q3. Where is the common landmark for knee } \\
\text { arthrocentesis to minimize trauma? }\end{array}$ & $37(66.1)$ & $29(49.2)$ & 0.067 \\
\hline $\begin{array}{l}\text { Q4. What is the depth of the needle that should be } \\
\text { advanced into the knee joint? }\end{array}$ & $39(69.6)$ & $33(55.9)$ & 0.129 \\
\hline $\begin{array}{l}\text { Q5. How should the needle be directed when advancing } \\
\text { into the knee joint? }\end{array}$ & $50(89.3)$ & $45(76.3)$ & 0.066 \\
\hline $\begin{array}{l}\text { Q6. How much volume should be aspirated provide } \\
\text { symptomatic relief of a large effusion? }\end{array}$ & $45(80.4)$ & $39(66.1)$ & 0.085 \\
\hline $\begin{array}{l}\text { Q7. Which type of specimen collection container should be } \\
\text { used? }\end{array}$ & $49(87.5)$ & $46(78.0)$ & 0.178 \\
\hline $\begin{array}{l}\text { Q8. What are the appropriate needle sizes (in gauge) for } \\
\text { the procedure? }\end{array}$ & $38(67.9)$ & $26(44.1)$ & 0.010 \\
\hline $\begin{array}{l}\text { Q9. After adequate aspiration of synovial fluid, which of the } \\
\text { following is the next most appropriate step before removing } \\
\text { the needle? }\end{array}$ & $46(82.1)$ & $36(61.0)$ & 0.012 \\
\hline $\begin{array}{l}\text { Q10. Which of the following general statement is correct } \\
\text { regarding knee arthrocentesis? }\end{array}$ & $47(83.9)$ & $42(71.2)$ & 0.103 \\
\hline
\end{tabular}

*Statistical significance was determined with Pearson Chi-squared test at $\alpha=0.05$

\section{Discussion}

Our study attempted to evaluate knowledge acquisition after learning a clinical skill via validated $M C Q$, which result illustrates that video-based online learning is more effective than text. The benefit of video can be justified by the dual-coding theory, suggesting the presence of both visual and auditory stimuli allows for more coherent pairing of imagery with its verbal counterpart (Clark and Paivio, 1991, Kanellopoulou et al., 2019). This bimodal method enables more effective encoding compared to a unimodal input (Clark and Paivio, 1991, Kanellopoulou et al., 2019). Likewise, the sensory semantic theory states that pictures can represent meaning more explicitly, which in turns, promotes memory formation (Nelson et al., 1977). These theories have been further explored in Worm and Jensen (Worm and Jensen, 2013), which they suggested modern online learning tools should be divided into levels according to its complexity as followed: Level 1 : text, images, and audio; Level 2: video and animation, and Level 3: complex animation with 3-dimensional graphics. Their study has shown that higher levels tools resulted in greater knowledge acquisition than lower level modalities (Worm and Jensen, 2013). This complements our finding that demonstrates significance intergroup differences in correct answer for questions involving visual cues (Table 1). Thus, students were more likely to remember specific equipment and anatomical landmark when they engaged in Level 2 video compared to Level 1 text passage.

Information bias was minimized by choosing a relatively short and simple procedure, with only 10 post-study MCQs to promote participant's engagement and compliance. Additionally, having five choices per question reduced false positive guesses. Since participants were instructed to study the content once without rereading, pausing, rewinding, or fast-forwarding the material, this minimized the confounding of individualized learning speed and repetition. 
While we are the first to date to perform an entirely online experiment simulating the online learning methods during the COVID-19 pandemic, this design is without its limitations. As controlling student's learning environment is a challenge for educator, the researcher also cannot oversee the experimental condition nor monitor the participant's adherence. Nevertheless, we attempted to address these shortcomings via randomization and recruitment of a sufficient sample size. As clinical proficiency requires acquisition of both knowledge and observable skills, we were only able to assess the 'knows' and 'knows how' via MCQ test, while technical competence was not examined (Thakker A, 2019). Although some have reported the use of virtual/online Objective Structured Clinical Examination (OSCE) for communication skills and history taking, the current technology may not feasibly assess clinical procedures, since they involve handson skills and require extensive equipment (Prettyman et al., 2018, Courteille et al., 2008).

\section{Conclusion}

Demonstration video is superior to text-based learning for knowledge acquisition of a procedural skill in Thai medical students. Therefore, the use of video tutorial should be encouraged during the COVID-19 online learning phase, to ensure optimal translation of theoretical knowledge to practical skills once clerkship resumes.

Acknowledgement: This research was supported by the Faculty of Medicine, Srinakharinwirot University.

Funding: Faculty of Medicine, Srinakharinwirot University, Thailand

\section{Conflicts of Interests: None declared}

\section{Authors' Contributions}

A.T. conceptualized the study. C.K., N.K., N.Y., S.T., and S.M designed the study and collected data. C.K., S.M. and A.T. performed the statistical analysis. All authors equally drafted, critically reviewed, and edited the manuscript.

\section{References}

Buch, S. V., Treschow, F. P., Svendsen, J. B. \& Worm, B. S. 2014. Video- or text-based elearning when teaching clinical procedures? A randomized controlled trial. Adv Med Educ Pract, $5,257-262$.

Clark, J. M. \& Paivio, A. 1991. Dual coding theory and education. Educ Psychol Rev, 3, 149-210.

Courteille, O., Bergin, R., Stockeld, D., Ponzer, S. \& Fors, U. 2008. The use of a virtual patient case in an OSCE-based exam--a pilot study. Med Teach, 30, e66-76.

Fitts PM, P. M. 1979. Human performance, Westport, Conn, Greenwood Press.

Kanellopoulou, C., Kermanidis \& Giannakoulopoulos, A. 2019. The Dual-Coding and Multimedia Learning Theories: Film Subtitles as a Vocabulary Teaching Tool. Educ Sci, 9, 210.

Komasawa, N., Terasaki, F., Nakano, T. \& Kawata, R. 2020. Relationships between objective structured clinical examination, computer-based testing, and clinical clerkship performance in Japanese medical students. PLoS One, 15, e0230792.

Miller, G. E. 1990. The assessment of clinical skills/competence/performance. Acad Med, 65, S63-7.

Nelson, D. L., Reed, V. S. \& Mcevoy, C. L. 1977. Learning to order pictures and words: A model of sensory and semantic encoding. J Exp Psychol Learn Mem Cogn, 3, 485-497.

O'doherty, D., Dromey, M., Lougheed, J., Hannigan, A., Last, J. \& Mcgrath, D. 2018. Barriers and solutions to online learning in medical education - an integrative review. BMC Med Educ, 18, 130.

Pai, K. M., Rao, K. R., Punja, D. \& Kamath, A. 2014. The effectiveness of self-directed learning (SDL) for teaching physiology to first-year medical students. Australas Medical J, 7, 448-453.

Prettyman, A. V., Knight, E. P. \& Allison, T. E. 2018. Objective Structured Clinical Examination From Virtually Anywhere! J Nurse Pract, 14, e157e163.

Reck-Burneo, C. A., Dingemans, A. J. M., Lane, V. A., Cooper, J., Levitt, M. A. \& Wood, R. J. 2018. The Impact of Manuscript Learning vs. Video Learning on a Surgeon's Confidence in Performing a Difficult Procedure. Front Surg, 5, 67.

Roy, B., Rashid, M., Sathian, B. \& Banerjee, I. 2018. Clinical skills and it's importance in undergraduate medical curriculum. J Biomed Sci, $4,1$. 
Sandhu, P. \& De Wolf, M. 2020. The impact of COVID-19 on the undergraduate medical curriculum. Med Educ Online, 25, 1764740.

Shaker, D. 2018. Cognitivism and psychomotor skills in surgical training: from theory to practice. Int $J$ Med Educ, 9, 253-254.

Taylor, J. A. \& Ivry, R. B. 2012. The role of strategies in motor learning. Ann N Y Acad Sci, 1251, 1-12.
Thakker A, D. P. 2019. Is there a role for virtual reality in objective structured clinical examinations (OSCEs)? MedEdPublish, 8, 31.

Worm, B. S. \& Jensen, K. 2013. Does peer learning or higher levels of e-learning improve learning abilities? A randomized controlled trial. Med Educ Online, 18, 21877 